\title{
Annotation
}

\section{Prevention of long term complications in diabetes}

\begin{abstract}
In September 1993 the Diabetes Control and Complications Trial (DCCT) reported their findings on the effect of improved glycaemic control on the microvascular complications of insulin dependent diabetes mellitus (IDDM). ${ }^{1}$ Taken in association with two other reports from independent sources in $1993^{23}$ there can now be little doubt that improved glycaemic control can positively effect the long term risks of developing nephropathy, retinopathy, and neuropathy.
\end{abstract}

\begin{abstract}
The trial
The DCCT represents a milestone in diabetes research. Altogether 1441 volunteers with IDDM between the ages of 13 and 39 years (approximately $20 \%$ in the 13 to 19 age range) were randomly assigned to receive either conventional once or twice daily insulin or a far more intensive regimen of three or more insulin injections or insulin pump treatment. In the conventionally treated group the aims were to avoid symptoms of hyperglycaemia, ketosis, and hypoglycaemia while maintaining normal growth and health. In the intensive group an attempt was made to achieve euglycaemia with a target preprandial blood glucose of between 3.9 and $6.7 \mathrm{mmol} / \mathrm{l}$ and postprandial concentrations of less than $10 \mathrm{mmol} /$; additionally glycated haemoglobin $A_{1 c}$ values were to be reduced to the normal range (below $6 \cdot 05 \%$ by the DCCT reference method). To achieve this end the intensive group visited a diabetic clinic every month, had frequent reviews by telephone, and 24 hour access to medical advice. Blood glucose was monitored at least four times a day and glycated haemoglobin measured every month. Insulin treatment was adjusted frequently according to both these results and the patient's alterations in diet and exercise.
\end{abstract}

\section{Results}

Each limb of the trial had two subsections according to the presence or absence of early diabetic microvascular complications: those with (the secondary intervention group) and those without (the primary prevention group) evidence of such disease. The average follow up period was 6.5 years and the drop out rate unusually low at $1 \%$, reflecting the extraordinary commitment of this study population of highly motivated volunteers. Intensive treatment reduced the mean glycated haemoglobin from $8.8 \%$ to $7 \cdot 2 \%$, only $5 \%$ of patients managing the target value of $6.05 \%$.

In the primary prevention group, intensive treatment reduced the adjusted mean risk of retinopathy (primary outcome variable for the study) by $76 \%$. Similarly the mean adjusted risk for microalbuminuria (the earliest manifestation of nephropathy) was reduced by $34 \%$ and for neuropathy by $69 \%$.

In the secondary intervention group the results were equally clear. Progression of retinopathy was reduced by $54 \%$ although, as has previously been described, there was an early phase of apparent worsening of retinopathy. ${ }^{4}$ The mean adjusted risk for microalbuminuria was reduced by $43 \%$ and for neuropathy by $57 \%$.
The major adverse side effect in the intensive study group was a far higher incidence of major hypoglycaemic events. Compared with the conventionally treated group in which there were 19 episodes per 100 patient years of hypoglycaemic events requiring a second person's intervention for treatment and five episodes per 100 patient years in which there was hypoglycaemic coma or seizure, the results for the intensive group were 62 and 16 per 100 patient years respectively.

A notable additional side effect of intensive treatment was a $33 \%$ increase in the mean adjusted risk of becoming overweight $(>120 \%$ of the ideal).

\section{Implications for care of children with diabetes}

It is notable that this trial included no children under the age of 13 years and we cannot assume that the same implications can be drawn for children before and after puberty.

\section{PREPUBERTAL CARE}

The DCCT population allows very few conclusions to be drawn about the treatment of children before puberty. Other studies have suggested that the contribution of the prepubertal years of diabetes on long term microvascular disease is minimal, ${ }^{56}$ however, this conclusion has recently been challenged. ${ }^{7}$ Profound hypoglycaemia in early childhood has serious implications for long term neurocognitive function. ${ }^{89}$ Consequently we should pause before striving for near normal glycaemic control in prepubertal children if this carries the risk of more frequent and serious hypoglycaemic episodes. Before puberty, in the absence of major technical advances in glycaemic control, we should aim for reasonable control, normal growth and development; additionally we should educate the children and their families about their disease in preparation for the need to institute a far more demanding approach in teenage. This view is succinctly put in an article by Allan Drash from Pittsburgh (a major contributor to the DCCT): 'although the philosophy of improved metabolic control should permeate all of our patients ... the specific goals of near metabolic normality should be reserved at this time for adolescence' ${ }^{10}$

\section{PUBERTAL CARE}

From the onset of puberty, children with diabetes are certainly at risk of glycaemia related microvascular disease. It is to this group that we must address the findings of the DCCT. There is no longer any excuse to fudge the issue of glycaemic control for adolescents: we now have incontrovertible evidence that glycaemic control is directly related to the development of microvascular disease. However, simply knowing that improving glycaemic control reduces the long term risk of developing serious microvascular disease only serves to restate the problem. Although we can see Nirvana, how do we get there?

It would be easy to attribute the improvement in glycaemic control in the DCCT to the more intensive 
insulin regimens used and simply adopt them for all adolescents. Unfortunately, however, a number of studies have shown that simply intensifying the insulin treatment in both children and adults has next to no effect on glycaemic control. $^{1112}$ A more reasonable conclusion is that the DCCT intensive regimen was effective in providing additional, frequent, at times daily support from medical and non-medical staff, in addition to the heightened personal involvement and commitment of the patients concerned. By its own admission the DCCT intensively treated group received far more attention and medical services than are routinely available in clinical practice in the USA. ${ }^{13}$

If we are to improve the glycaemic control of adolescents with diabetes it will not be enough simply to change insulin regimens. Firstly, we have to convince teenagers that more blood tests, clinic visits, and medical advice will be beneficial to them in the long term. Although the DCCT reported no particular problems with the 13-18 age group, they were highly selected and specifically motivated volunteers (not obviously representative of adolescents attending clinics in the UK!).

Secondly, if we are to provide intensive support we have to convince the financiers of diabetes health care that additional medical, nursing, and paramedical input is essential if long term diabetes control is to be improved and sustained. Are the purchasers likely to fund many more diabetes clinics, with the additional input needed from medical and support staff, notably specialist diabetes health visitors and behavioural psychologists? Improvements in staff/patient communication will require 24 hour telephone advice including weekends and holiday and cross boundary cover for essential staff such as health visitors and dietitians.

If and when additional staff are trained and appointed there is then, among adolescents, a case for seeking stricter glycaemic control with increased patient involvement in blood testing and alterations in insulin treatment. The introduction of a more intensive insulin regimen, three or more injections per day or a return to insulin pumps would then have to be subject to new field trials to evaluate acceptability, efficacy, and side effects.

Even with a huge increase in clinical commitment, however, there are likely to be significant numbers of teenagers who cannot or will not take up the gauntlet of improved glycaemic control with its attendant risks of increased hypoglycaemic events. As Stephanie Amiel pointed out in a recent $B M \mathcal{F}$ editorial: many young people would worry more about losing their driving licences as a result of severe hypoglycaemia than they would about the later prospect of renal failure. ${ }^{14}$ It is unfortunate that a large number of those who would benefit most from an improvement in glycaemic control, being the worst controlled at present, will be among this group because at the extremes of poor control the DCCT indicates escalating risks of diabetes complications. At least one centre has already taken up this particular challenge and by employing skilled counsellors together with a great investment of time have secured at least some improvement in medium term control in such a teenage group (R Newton, S A Greene (Dundee); personal communication).

\section{Conclusions}

In children under 10 years it is questionable whether there is a case for tightening of glycaemic control through the intensification of treatment, with its attendant risks of increasing the frequency of serious hypoglycaemic events. However, it must now be the responsibility of the clinical team to ensure that by the age of 10 years, the child and family have been prepared for the intensification of attempts to achieve normoglycaemia at the onset of puberty.

Once the decision to intensify treatment has been taken it must be recognised that this does not simply amount to increasing the frequency of insulin administration. The imposition of more demanding insulin regimens and the counterproductive effects this may have on teenage compliance dictate that the question as to the best regimen to be used in a typical teenage group has to be resolved by further trials. Many teenagers may simply be unwilling to follow demanding treatment programmes. For instance, teenage girls are unlikely to accept a regimen that is more likely to cause excess weight gain as was seen in the DCCT intensive treatment group. For this reason we should not be blind to other potential therapeutic interventions, each of which demands introduction only as part of a therapeutic trial. Such an intervention is the proposed use of angiotensin converting enzyme inhibitors in young patients with incipient nephropathy.

Taking the DCCT results at face value, we are in danger of launching a therapeutic panacea for young people with diabetes, understandably motivated by our wish to reduce the appalling burden of microvascular disease in later life. We would be better advised, however, to take the opportunity of the DCCT challenge and seek to re-evaluate and restructure. Now the DCCT genie is out of the bottle it cannot be ignored. Coupled with the demands from the St Vincent declaration, ${ }^{15}$ our task must become more onerous, demanding, and responsible. It will mean increased time, staff, money and, most importantly, commitment.

\section{J P. H SHIELD} J D BAUM

Institute of Child Health,

Royal Hospital for Sick Children

St Michael's Hill,

Bristol BS2 8Bf

1 The Diabetes Control and Complications Trial Research Group. The effect of intensive treatment of diabetes on the development and progression of long-term complications in insulin-dependent diabetes mellitus. $N$ Engl $f$ Med 1993; 329: 977-85.

2 Wang PH, Lau J, Chalmers TC. Meta-analysis of effects of intensive blood glucose control on late complications of type 1 diabetes. Lancet 1993; 341: 1306-9.

3 Reichard P, Nilsson B-Y, Rosenqvist U. The effect of long-term intensified insulin treatment on the development of microvascular complications of diabetes mellitus. N Engl F Med 1993; 329: 304-9.

4 Lauritzen T, Frost-Larsen K, Larsen HW, Deckert T. Effect of 1 year of near-normal blood glucose levels on retinopathy in insulin-dependent diabetics. Lancet 1983; i: $200-4$

5 Kostraba JN, Dorman JS, Orchard TJ, et al. Contribution of diabetes duration before puberty to the development of microvascular complications in IDDM subjects. Diabetes Care 1989; 12: 686-93.

6 Burger W, Hovener G, Dusterhus R, Hartmenn R, Weber B. Prevalence and development of retinopathy in children and adolescents with type 1 (insulin-dependent) diabetes mellitus. A longitudinal study. Diabetologia 1986; 29: 17-22.

7 McNally PG, Raymond NT, Swift PGE, Hearnshaw JR, Burden AC. Does pre-pubertal duration of diabetes influence the onset of microvascular pre-pubertal duration of diabetes influence the
complications? Diabetic Med 1993; 19: 906-8.

8 Ryan C, Vega A, Drash A. Cognitive deficits in adolescents who developed diabetes early in life. Pediatrics 1985; 75: 921-7.

9 Rovet JF, Ehrlich RM, Hoppe M. Intellectual deficits associated with early onset insulin dependent diabetes mellitus in children. Diabetes Care 1987; 10: $510-5$.

10 Drash AL. The child, the adolescent, and the Diabetes Control and Complications Trial. Diabetes Care 1993; 16: 1515-6.

11 Mortensen HB, Villumsen J, Volund A, et al. Relationship between insulin injection regimen and metabolic control in young Danish type 1 diabetic patients. Diabetic Med 1992; 9: 834-9.

12 Bougneres PF, Jos J, Garandeau P, et al. Improvement of diabetic control and acceptability of a three-injection insulin regimen in diabetic adolescents. Diabetes Care 1993; 16: 94-102.

13 Santiago JV. Lessons from the Diabetes Control and Complications Trial. Diabetes 1993; 42: 1549-54.

14 Amiel S. Diabetic control and complications. BMf 1993; 307: 881-2.

15 WHO/IDF Europe. Diabetes care and research in Europe: the St Vincent declaration. Diabetic Med 1990; 7: 360. 